\title{
Effect of life style abnormality in adolescent age group
}

\author{
Available online at www.ijistweb.com
}

RESEARCH ARTICLE

Aashutosh, Dinesh Kumar*

Department of Biochemistry, Sri Satya Sai University of Technology \& Medical Sciences, Sehore-466001

*Corresponding Author's E-mail:om11agra85@gmail.com

\begin{abstract}
A complete urinalysis includes physical, chemical and microscopic examinations. Midstream clean catch collection is acceptable in most situations, but the specimen should be examined within two hours of collection. Cloudy urine often is a result of precipitated phosphate crystals in alkaline urine, but pyuria also can be the cause. A strong odour may be the result of a concentrated specimen rather than a urinary tract infection. Dipstick urinalysis is convenient, but false-positive and false-negative results can occur. Specific gravity provides a reliable assessment of the patient's hydration status. Glomerular, renal and urologic causes of microhaematuria often can be differentiated by other elements of the urinalysis. Although transient proteinuria typically is a benign condition, persistent proteinuria requires further work-up.
\end{abstract}

Keywords: Adolescent, Urinalysis, Study, Life Style.

\section{Introduction}

Adolescents have 2/3 of total population. This is a unique group with special needs and attention. Most adolescents with lifestyle issues fell in the age group of 16-18 years. Adolescence is an important transition in an individual's life. Turmoil which one experiences in this period-has greater effects onto the behavioral aspects of an individual. If one divides the whole stream of an individual's life into certain urbitrary segments, then adolescence is that period of life which lies between childhood and adulthood (1-4). Although there is no definite day or hour when childhood ends and adolescence begins, it has become conventional to fix upon the first signs of reproductive power as the beginning of adolescence. But the age of the onset of such reproductive power (popularly known as 'puberty') varies with the climates, with race, with intelligence, and with some such other conditions (5-8).

It is standard methods of adherence monitoring for prescribed substances, for example, self-reporting and monitoring of symptoms or patient behaviors, are unreliable for controlled substances. Though volumes have been made available by the previous researchers on several aspects of adolescents problem, still the 'problems of adolescence as such is quite novel even in the present era and that is because of its crucial and delicate impact on to the individual as well as to the society (9-12).

Studies so far reported on various problems of adolescence in the country and abroad cover a wide range starting from problems of adjustment in general (13-14), to the problems of adjustment in context to cross-cultural variations (15), in rural and urban adolescents (16), in context to personality and motivational aspects of adolescents (17-18).

A scrutiny of these studies gives an impression 'that there is as yet lack of agreement amongst these authors regarding the onset as well as duration of adolescence in an individual's life. This is so stated because in some' of these studies the subjects (Ss) selected were in the age range of 14 to 18 years, in some others 15 to 17 and in some others the beginning of adolescence was thought to be as early as 12 years, and still in some others no mention of the age of the subjects was made but that they were all undergraduate students (19). In none of these studies any justification for selecting subjects within this age range was mentioned. Perhaps following Western 
literature it has been assumed in these studies that the classical age range of adolescence is between 14 to 18 years. If this assumption is true, then certainly it is subject to criticism since there are wide cross-cultural and sub-cultural 0 variations in the onset of adolescence (20). Thus, e.g., for the girls of Western cultures the beginning of adolescence has been reported to vary between age 9 to 20 years or even later depending upon whether she is Italian, English or Finns (21).

An important consequence of our modern-day society is that it is difficult for children and caregivers both to get adequate sleep. Sleep involves reciprocal interactions between all members of a household as well as with the environment of the home and outside.

\section{Materials and Methods}

Drugs, chemicals and other substances consumed by humans are often excreted in the urine, where they may be detected with screening tests. The detection of these compounds is limited only by the assay and cost.

\section{Forensic versus clinical urinary drug testing}

Historically drug testing has been forensic in nature and has assumed most donors will provide a negative specimen. In patient-centered UDT in a clinical setting, the majority of specimens provided are expected to be positive for a broad range of drugs that are prescribed for medical purposes which adds to the complexity of the testing and the interpretation of data. Most UDT today that involves drug testing laboratories includes elements of both forensic drug testing and clinical drug testing. Drug testing in clinical settings also includes toxicology testing, usually in hospital emergency departments or emergency psychiatry settings, used to help accurately diagnose possible drug poisoning or overdose. Clinical drug testing is often inaccurately labeled as "toxicology testing" involving "tox screens" when the goal of testing is not to identify a case of acute poisoning but is to assist in treatment planning for a chronic disease, such as chronic non cancer pain or addiction.

In forensic drug testing, results are meant to stand up to legal challenges and meet the rules of evidence in legal proceedings. Chain-of-custody procedures, secure storage of samples, and stringent method validations are utilized with the aim of minimizing or eliminating false positive results, and rigorous laboratory certification programs are used to assure quality. The personnel running the tests in a forensic UDT laboratory usually have training in chemistry or forensic science and they understand chain-ofcustody and medicolegal requirements In hospital or pathology laboratories the emphasis is typically on drugs of abuse, screening either for compliance to (e.g. methadone) or abstinence from (e.g. cannabis) particular substances. Hospital screening can also give the clinician additional help in the differential diagnosis and treatment of a patient, but the availability of toxicological analyses varies between hospitals.

Most commonly used drug screening tests involve immunoassay techniques. These range from so-called bedside or point-of-care testing to more sophisticated laboratory based immunological tests. Immunoassay can provide fast and reliable results, however the results must be interpreted with caution.

\section{Results and Discussion}

Urine drug test in clinical practice, like any other medical test, should be performed to improve patient care. Inappropriate interpretation of results, as with any other diagnostic test, may adversely affect patient care: for example, discharge of patients from care when prescribed drugs are not detected and over- or underdiagnosis of substance misuse or addiction. Health care professionals should use UDT results in conjunction with other clinical information. Consultation with an individual knowledgeable in UDT interpretation (eg.: laboratory director or toxicologist) is strongly encouraged, especially when unexpected test results are obtained. The testing laboratory or POC device manufacturer should provide readily accessible consultation and results interpretation.

\section{Interpretation of UDT results}

The valid detection period for drug exposure varies depending on the disposition characteristics 
of the drug, dose, and frequency of use. Specific characteristics of a urine sample include its appearance, temperature within 4 minutes of voiding, $\mathrm{pH}$, creatinine concentration, and specific gravity. The color of urine is based on the concentration of its constituents and can vary based on medications, foods, or disease states; excess hydration can cause it to appear colorless. Concentrated urine specimens are usually more reliable than dilute specimens.

Table 1 Interpretation of urine drug test

\begin{tabular}{|lll|}
\hline Positive test result & Patient has taken drug & Patient has not taken drug \\
\hline Negative test result & False negative & False Positive \\
\hline
\end{tabular}

In this context, the sensitivity of a test is the ability to detect a class of drugs, while the specificity is the ability to identify a particular drug. A highly specific test gives few falsepositive results and identifies individual drugs and/or their metabolites. High sensitivity is due, in part, to the test's ability to detect both the parent drug and/or its metabolite(s), combined, to reach the cutoff concentration for a positive report.

\section{Sensitivity and specificity}

The qualitative immunoassay drug panel reports each sample as either positive or negative for a particular drug or drug class, based on predetermined cutoff concentrations. In the ideal world, UDT would be positive if the patient took the drug (true-positive) and negative if the drug was not taken (true-negative). However, falsepositive or false-negative results can occur, so it is imperative to interpret UDT results carefully. In addition, testing technology is constantly evolving and varies by manufacturer, so false-positive or false-negative results today may not be relevant in the future.

\section{Cross-Reactivity}

Detection of a particular drug by a drug-classspecific immunoassay depends on the structural similarity of that drug or its metabolite(s) to the compound used for standardization, and the urine concentration of that drug/metabolite, compared with the standardizing compound.9 For example:

Tests for cocaine react principally with cocaine and its primary metabolite, benzoylecgonine.
These tests have low crossreactivity with other substances and, therefore, presence of cocaine (detected only with very recent use because of a short half-life) or benzoylecgonine is highly predictive of cocaine use.

Tests for amphetamine/methamphetamine are highly crossreactive. They may detect other sympathomimetic amines such as ephedrine and pseudoephedrine and, therefore, are less reliable for amphetamine/methamphetamine use. Further testing may be required by a more specific method, such as GC/MS and stereospecific chromatography.

Immunoassay testing for opiates is very responsive for morphine and codeine, but does not distinguish which is present. However, it shows a lower sensitivity for semisynthetic/ synthetic opioids, and so even large concentrations in the urine may not be reliably detected by the opiate immunoassay. A negative result does not exclude use of these opioids, but the ability of opiate immunoassays to detect semisynthetic/synthetic opioids varies among assays because of differing cross-reactivity patterns. Specific immunoassay tests for some semisynthetic/ synthetic opioids may be available (eg, oxycodone, methadone/EDDP).

Therefore, for clinical purposes, the cocaine assay would be very reliable, while the amphetamine assay would be unreliable in predicting use of the drug, and the opiate assay would be unreliable in predicting use of semisynthetic/synthetic opioids. Fortunately, GC/MS or LC/MS analysis directed toward a 
particular molecule on the same urine specimen will normally detect these semisynthetic and synthetic opioids. It is important to contact the laboratory when looking for a specific substance to ensure that the correct test/profile is used.

Cross-reacting compounds can also be structurally unrelated to the standardizing compound. For example, several quinolone antibiotics (eg, levofloxacin, ofloxacin) can potentially cause false-positive results for opiates by some common immunoassays, despite no obvious structural similarity with morphine. Quinolones are not misidentified as opiates by

Table 2 Examples of cross-reacting compounds for certain immunoassays

\begin{tabular}{|ll|}
\hline \multicolumn{1}{|c|}{ Interfering drug } & Immunoassay Interfering drug affected \\
\hline Quinolone antibiotics(eg, levofloxacin, ofloxacin) & Opiates \\
\hline Antidepressant trazodone & Fentanyl \\
\hline Antidepressant venlafaxine & Phencyclidine \\
\hline Atypical antipsychotic quetiapine & Methadone \\
\hline Antiretroviral efavirenz & THC \\
\hline Diet pills (eg, clobenzorex, fenproporex) & Amphetamine \\
\hline $\begin{array}{l}\text { Promethazine (for allergies, agitation, nausea, } \\
\text { vomiting) }\end{array}$ & Amphetamine \\
\hline l-methamphetamine (over-the-counter nasal inhaler) & Amphetamine \\
\hline Dextromethorphan & Phencyclidine \\
\hline Proton pump inhibitors (such as pantoprazole) & THC \\
\hline
\end{tabular}

\section{Drug Metabolites}

In general, the concentration of the parent drug in urine exceeds that of its metabolite(s). In certain cases, UDT may detect traces of unexplained opioids (Figure 4.1) For example, a patient who is prescribed codeine may show trace quantities of hydrocodone that may not represent hydrocodone use. Detection of minor amounts of hydrocodone in urine containing a high concentration of codeine should not be interpreted as evidence of hydrocodone use. In the case of a patient who is prescribed hydrocodone, quantities of hydromorphone may be detected because of hydrocodone metabolism. However, the detection of trace amounts of a potential metabolite in the absence of its parent may be a timing of administration issue rather than coadministration of a second drug. As with any unexplained test result, it is important to clarify the interpretation
GC/MS or LC/MS. There have also been cases of false-positive fentanyl results with some immunoassays for patients who are taking the antidepressant trazodone and the antidepressant venlafaxine can cause false-positive PCP results with some immunoassays. Examples of other agents that can cause false-positive immunoassay results are shown in Table 4.2. Interferences from some of the drugs listed have been eliminated by some manufacturers, and other interferences are expected to arise as tests are modified and new drugs come to market. 
Codeine metabolizes to morphine, but the reverse does not occur.

Codeine alone is possible because a small proportion of patients (7\% of the Caucasian population) lack the necessary activity of the cytochrome P450 (CYP) 2D6 enzymatic pathway to convert codeine to morphine.

Metabolism of codeine would not result in the presence of hydromorphone.

Morphine may be metabolized to produce small amounts (generally <10\%) of hydromorphone.

Hydrocodone may be metabolized to small quantities of hydromorphone.

Metabolism of codeine may produce small quantities of hydrocodone.

Oxycodone is metabolized by CYP3A4 to noroxycodone and by CYP2D6 to oxymorphone. If the urine of a patient prescribed oxycodone tests positive for oxymorphone, a quantitative analysis should confirm - in the majority of cases - that the relative concentration of oxycodone is greater than oxymorphone. Test results for patients prescribed oxymorphone are easier to interpret because oxymorphone does not produce any metabolites that can be mistaken for another opioid (although oxymorphone tablets may contain up to $1 \%$ oxycodone as a manufacturing byproduct, which should generally not be detectable with UDT).

Cocaine: Cocaine is a topical anesthetic clinically used in certain trauma, dental, ophthalmologic, and otolaryngologic procedures. A patient's urine may test positive for the cocaine metabolite, benzoylecgonine, after such a procedure for up to 2 to 3 days. However, a licensed health care professional must order its use, which can be checked through medical records or by contacting the treating health care professional. There is no structural similarity between other topical anesthetics that end in "caine" (eg, prilocaine, lidocaine) and cocaine or benzoylecgonine; therefore, cross-reaction does not occur.A positive UDT result for the cocaine metabolite, in the absence of a medical explanation, should be interpreted as due to deliberate use.
Clinical interpretation of positive amphetamine and methamphetamine results can be challenging because of the structural similarities to many prescription and OTC products, including diet agents, decongestants, and selegiline used in the treatment of Parkinsons disease. Knowledge of potential sources of amphetamine and methamphetamine can prevent misinterpretation of results. The traditional GC/MS criteria for reporting a positive methamphetamine result is not sufficient to distinguish methamphetamine use from use of OTC products. Methamphetamine exists as 2 isomers that are designated $\mathrm{d}$ - and 1-.8 The d-form has a strong stimulant effect on the central nervous system (CNS) and high misuse potential, while the 1-form in therapeutic doses has a primarily peripheral action and is found in some OTC preparations. Routine testing, such as immunoassays or GC/MS, does not differentiate between the d- and 1-forms. In a case of disputed amphetamine or methamphetamine misuse, stereospecific chromatography may be used in addition to GC/MS. For example, the OTC Vicks® Inhaler marketed in the United States contains 1desoxyephedrine (l-methamphetamine).8 Patients whose management includes UDT should be advised not to use the Vicks® Inhaler or similar OTC preparations containing this agent because they can interfere with the interpretation of UDT results; this is particularly important in a community with a high incidence of methamphetamine misuse. Misuse of even the 1form can have significant CNS activity and should be addressed clinically with the patient.

\section{Interpretation of results}

Clinicians' predictions of UDT results are often inaccurate and evidence suggests a majority of physicians have a poor understanding of how to interpret UDT results. Others may have a false sense of confidence about interpreting their patients' UDT results because they lack specific knowledge or don't fully understand the breadth of abnormal or unexpected toxicology findings that are possible

\section{Conclusion}

Amphetamine/Methamphetamine: 
Adolescents need to be treated as a distinct segment of our population and it is important to realize and address their health and lifestyle problems. Urine drug testing can be an effective tool for health care professionals in the assessment and ongoing management of patients who:

Have or may have the disease of addiction

Have other relevant medical conditions or diagnoses

Will be, or are being, treated over the long term with controlled substances, including opioids (not for acute pain)

The demands on young people are new and unprecedented; their parents could not have predicted many of the pressures they face. How we help adolescents meet these demands and equip them with the kind of education, skills, and outlook they will need in a changing environment will depend on how well we understand their world. Because substance-use disorders are common, UDT should be considered a core tool in primary care. The clinician can use a discordant UDT result to motivate patient behavior change. However, testing without an appropriate strategy for interpreting results can do harm. A working relationship with the testing laboratory or test kit provider is essential to accurately interpret UDT results. Most importantly, a health care professional should strive for a relationship of mutual honesty and trust with the patient when using UDT in his or her clinical practice. Therefore, the use of UDT should be consensual, be designed to help patients, and assist health care professionals to advocate for the health and wellbeing of their patients. Further research studies should be carried out to highlight issues of concern and their possible solutions in this population

\section{Acknowledgement}

The authors are thankful to IJIST Journal for publishing their article.

\section{Conflicts of Interest}

The author declares that there are no conflicts of interest.

\section{References}

1. Trager W., and Jenson J.B... Cultivation of malarial parasites. Nature, 1978; 273:621-622.CrossRefMedline.

2. Waller R. F., and McFadden GI. The apicoplast: a review of the derived plastid of apicomplexan parasites. Curr. Issues Mol. Biol. 2005; 7:57-79.

3. Waller R. F., Reed MB, A. Cowman AF, McFadden GI. Protein trafficking to the plastid of Plasmodium falciparum is via the secretory pathway. EMBO J. 2000; 19:1794-1802.CrossRefMedline

4. Roberts A., Trapnell C., Donaghey J., Rinn J.L. and Pachter, L.. Improving RNASeq expression estimates by correcting for fragment bias. Genome Biol 2011; $12: \mathrm{R} 22$.

5. Roberts, L. \& Enserink, M. Did they really say.. eradication? Science 2007; 318:1544-1545.

6. Roos V., Nielsen E.M. and Klemm P. Asymptomatic bacteriuria Escherichia coli strains: adhesins, growth and competition. FEMS Microbiol Lett 2006; 262:22-30.

7. GuptaS., ThaparM. M., MarigaS. T., WernsdorferW. H., BjorkmanA. Plasmodium falciparum: in vitro interactions of artemisinin with amodiaquine, pyronaridine, and chloroquine. Exp. Parasitol. 2002; 100:28-35.CrossRef

8. He C. Y., M. K. Shaw C. H. Pletcher, B. Striepen, L. G. Tilney, and D. S. Roos. A plastid segregation defect in the protozoan parasite Toxoplasma gondii. EMBO J. 2001; 20:330-339.CrossRefMedline

9. Hemmer C. J. et al. Stronger host response per parasitized erythrocyte in Plasmodium vivax or ovale, than in Plasmodium falciparum malaria. Trop. Med. Intl Health 2006; 11:817-823.

10. Fichera M. E., M. K. Bhopale and D. S. Roos. In vitro assays elucidate peculiar kinetics of clindamycin action against Toxoplasma gondii. Antimicrob. Agents Chemother. 1995; 39:1530-1537.Abstract/FREE Full Text

11. Foth B. J., S. A. Ralph C. J. Tonkin, N. S. Struck, M. Fraunholz, D. S. Roos, A. F. Cowman, and G. I. McFadden. Dissecting apicoplast targeting in the malaria parasite Plasmodium falciparum. Science, 2003; 299:705-708. Abstract/FREE Full Text

12. Frisk-HolmbergM., BergqvistY., TermondE., DomeijNybergB. The single dose kinetics of chloroquine and its major metabolite desethylchloroquine in healthy subjects. Eur. J. Clin. Pharmacol. 1984; 26:521530.CrossRefMedline

13. Cook J. A., E. J. Randinitis C. R. Bramson, and D. L. Wesche. Lack of a pharmacokinetic interaction between azithromycin and chloroquine. Am. J. Trop. Med. Hyg. 2006; 74:407-412. Abstract/FREE Full Text

14. Cox-Singh, J. et al. Plasmodium knowlesi malaria in humans is widely distributed and potentially life threatening. Clin. Infect. Dis. 2008; 46:165-171.

15. Borrmann S., S. Issifou G. Esser A. A. Adegnika, M. Ramharter, P. B. Matsiegui, S. Oyakhirome, D. P. Mawili-Mboumba, M. A. Missinou, J. F. Kun, H. Jomaa, and P. G. Kremsner. Fosmidomycin-clindamycin for the treatment of Plasmodium falciparum malaria. $\mathbf{J}$. 
Infect. Dis. 2004; 190:1534-1540.Abstract/FREE Full Text

16. Boyle J. The effect of the high school on students' aspirations. Amer. J. Sociol., 71 , 628-639.

17. McConkey G. A., M. J. Rogers and T. F. McCutchan. Inhibition of Plasmodium falciparum protein synthesis. Targeting the plastid-like organelle with thiostrepton. J. Biol. Chem. 1997; 272:2046-2049.Abstract/FREE Full Text

18. Medline GustafssonL. L., et al. Disposition of chloroquine in man after single intravenous and oral doses. Br. J. Clin. Pharmacol. 1983; 15:471-479.2006.
19. Meshnick S. R., Taylor T. E. \& Kamchonwongpaisan, S. Artemisinin and the antimalarial endoperoxides: from herbal remedy to targeted chemotherapy. Microbiol. Rev. 1996; 60:301-315.

20. Roos V., Nielsen, E.M. and Klemm, P. Asymptomatic bacteriuria Escherichia coli strains: adhesins, growth and competition. FEMS Microbiol Lett 2006; 262:22-30.

21. Rosenberger C.M. and Finlay, B.B. Phagocyte sabotage: disruption of macrophagesignalling by bacterial pathogens. Nature reviews. Molecular cell biology 2003; 4:385-396. 